\title{
Long Term Outcomes of Cross-Hatching Eustachian Tuboplasty
}

\author{
Carlos Yanez, Sandra Velázquez, Nallely Mora \\ American British Medical Center, Mexico City, Mexico \\ Email: carlosyanez md@yahoo.com
}

Received 24 October 2014; revised 17 November 2014; accepted 30 November 2014

Copyright (C) 2015 by author and Scientific Research Publishing Inc.

This work is licensed under the Creative Commons Attribution International License (CC BY). http://creativecommons.org/licenses/by/4.0/

(c) (i) Open Access

\begin{abstract}
Objective: To review the long term outcomes of cross-hatching Eustachian Tuboplasty (ChEt) in patients with chronic obstructive Eustachian tube dysfunction (COETD), as well as assess the clinical factors associated with surgical success. Study Design: Retrospective case series review. Setting: Tertiary healthcare institution. Methods: This is a retrospective review of non-revision ChET for COETD. Follow-up period was 5 years. The inclusion criteria were persistent otitis media with effusion, conductive hearing loss of 5 or more years, and constant COETD related-symptoms. The curvature of the posterior cushion was modified using an argon laser to alter the spring of the cartilage alleviating the obstructed valve's aperture. Several clinical factors were reviewed in relation to the successful opening of Eustachian tube valve. Results: One hundred and twenty patients, $\mathbf{7 2}$ males/48 females, average age $42.4+2$ years old, met study inclusion criteria. COETD patients/ obstructive causes were: Posterior cushion hypertrophy, 68 (56.6\%); Tensor Veli and Levator Veli Palatini muscles hypertrophy, 15 (12.5\%); Remarkable mucosal hypertrophic disease, 37 (30.8\%). Total of ET tubes was 198. Bilateral $143(72.2 \%)$, 55 unilateral $(27.7 \%)$ ET valve was seen more open postoperatively on simple endoscopy (SE) and slow motion video analysis (SMVEA). There were no complications. Mean pure tone average improved by $20 \mathrm{~dB}$ postoperatively; $P=0.015$. Mean immitance changes in tympanometric measurements improved postoperatively at least 0.10 mmhos in $91 \%$ of the patients $(P=0.010)$. Resolution of symptoms was considered a successful outcome. Failure correlated with the severity of disease. Conclusion: High rates of improvement (96\%) were achieved. ChEt is a promising technique for the treatment of COETD.
\end{abstract}

\section{Keywords}

Obstructive Eustachian Tube Dysfunction, Eustachian Tuboplasty

\section{Introduction}

Chronic obstructive Eustachian tube dysfunction (COETD) is a common disorder causing repeated visits to the

How to cite this paper: Yanez, C., Velázquez, S. and Mora, N. (2015) Long Term Outcomes of Cross-Hatching Eustachian Tuboplasty. International Journal of Otolaryngology and Head \& Neck Surgery, 4, 6-12. 
doctor's office and substantial medical expenses. The most common symptoms are autophony, hypoacusia, tinnitus and fullness of the ear. When medical treatment fails, surgery should be considered an option. Tympanostomy tube placement is the common procedure. Multiple insertions of ventilating tubes are required if refractory otitis media persists. Numerous surgical procedures have been proposed for correcting COETD. Hopf et al. [1] proposed that intraluminal surgery improves Eustachian tube dysfunction using laser techniques. Kujawski [2] [3] performed the first in 1997 using a $\mathrm{CO}_{2}$ laser focused on the cartilaginous portion and reported that $81 \%$ of his 38 patients were symptom-free after 36 months of follow-up. Poe, Metson and Kujawski [4] describe their preliminary results on Laser Eustachian Tuboplasty on 2003. We [5] described the laser-assisted cross-hatching Eustachian Tuboplasty (ChEt) and reported the preliminary results in 25 patients including a 15-month followup. Overall results showed that $92 \%$ were free of middle ear effusion with symptoms improving. Nowadays, laser-assisted ChEt is being considered a minimally invasive surgical modality for COETD, although further studies are required for determining whether it is alternative to COETD, instead of repeated tympanostomy tube placement in selected patients. We also developed a Eustachian tube dysfunction numerical staging, as well as results reporting system that better assists in the analysis of Eustachian tube surgery [6]. We present here the outcomes of ET surgery in our personal clinical patient series.

\section{Materials and Methods}

This is a retrospective case series review carried out at the Sinus Surgery Center, inside a Tertiary Care Hospital, the American British Cowdray Medical Center, located in Mexico City, where 120 consecutive patients with COETD were diagnosed between February 2001 and June 2008. Patients were classified according to the Yanez Classification System [6], surgically treated with ChET and followed for 5 years postoperatively. The inclusion criteria were persistent otitis media with effusion, with a history of multiple tympanostomy tube insertions; conductive hearing loss of 5 or more years, and constant COETD related-symptoms. Age and gender were equally distributed. Exclusion criteria were allergic rhinitis, laryngopharyngeal reflux and other type of otologic or nasal surgery. We included 72 males and 48 females, mean age $42.4 \pm 2$ years, the youngest being 4 and the oldest 60 years old. They served as their own controls. All patients provided informed consent for the surgical procedure and the study was reviewed and approved by the Ethics Committee of the Sinus Surgery Center.

For the classification system, patients were studied as following:

1) Axial and coronal computer tomography scans of the ear were taken for measuring the width of the bony portion of the Eustachian tube (ET) and the cartilaginous portion morphology, studied while the patients performed the Valsalva maneuver, as well as identifying the internal carotid artery and the relationship with the ET. The radiological pathology of the cartilaginous portion of the ET was classified based on mucosal intraluminal swelling as normal, or swollen/opacified; done by a Radiologist that doesn't know the patients.

2) Preoperative symptoms (earache, hearing loss, ear fullness, tinnitus, autophony and vertigo) were recorded using a 3-point scale ( 0 = absent; 1 = mild; 2 = moderate; 3 = severe symptom).

3) All patients underwent a trans-nasal endoscopic slow motion video analysis (SMVEA) of their Eustachian tubes, done with a $30^{\circ}$ view angle using a rigid Hopkins rod endoscope measuring $4.0 \mathrm{~mm}$ or $2.7 \mathrm{~mm}$ diameter, (Karl Storz, Tuttinghem, Germany), recorded and analyzed on a S-VHS/SR-VCR in normal time, in slow motion and single frame viewing at 30 frames per second. Examinations were performed while the patient was awake and administered topical anesthesia by placing two or three cotton swabs soaked in a $2 \%$ Pontocaine solution and left for 5 to 7 minutes in the inferior meatus. The endoscopes were directed into the pharyngeal orifice while the patient remained sitting and rested his/her head in the chair's headrest. The entire nose was first inspected and the $30^{\circ}$ angle view was directed laterally. The ET medial structures and valve were examined. Subjects were asked to repeat the letter $\mathrm{K}$, to swallow and to yawn for better viewing of the palate, medial cartilaginous lamina, tensor veli palatine muscle (TVPM), levatorveli palatine muscle (LVPM), sphenopharyngeousmuscle (SphM) and record valve dynamics in both normal and forced motion modes. Figure 1 shows the phases of valve dilation in a normal Eustachian tube valve.

4) All patients underwent auditory battery tests (pure tone audiometry, tympanometry, Eustachian tubetympanometric tests).

5) Patients were classified following the staging system used to identify the extent of the surgical procedure required for every patient. 
SLOW MOTION VIDEO ENDOSCOPY PHASES OF VALVE DILATION
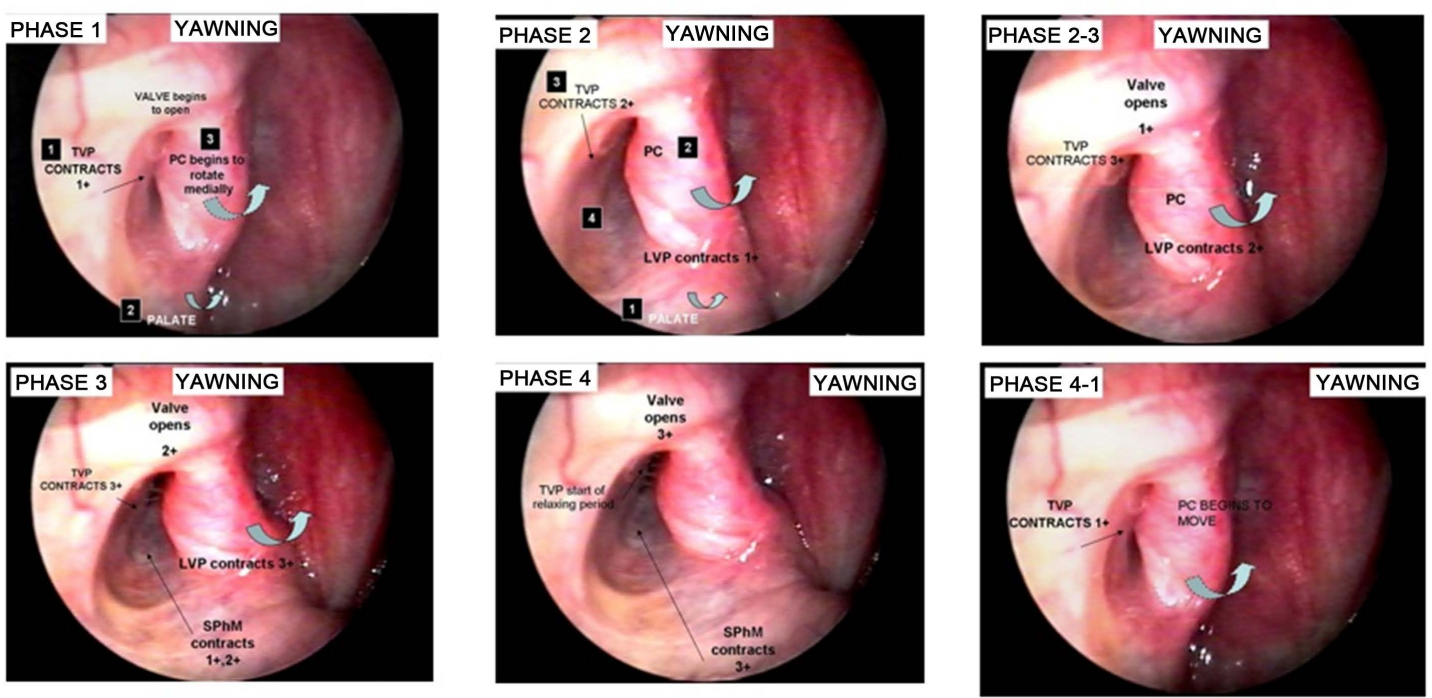

Figure 1. Slow-motion video-endoscopic analysis. We can see the phases of valve dilation that are useful to classify the Eustachian tube dysfunction.

\subsection{Laser Eustachian Tuboplasty Procedure-Cross-Hatching}

All patients were operated under general anesthesia by the senior author of this article. The ChEt procedure included a bilateral diagnostic nasal endoscopy carried out under general anesthesia. Special attention was paid to examining the ET valve, TVPM, LVPM and SphM areas. Mucosal contact areas, polypoid or granulomatous tissue were studied in detail. Anatomical variations, such as altered cartilaginous spring of the posterior cushion, abnormally wide or thick posterior cushion, valve morphology, pronounced superior direction of the lumen and valve or absence of the posterior cushion cartilaginous super-structure (congenital or iatrogenic) were also noted and recorded. Obstructive and/or hypertrophic areas of the tube were confirmed by instrument palpation and direct visualization and its direct impact to the valve area was recorded. A tonsil mouth gag was inserted and the mouth opened.

Instruments were placed in a combined fashion using both the endonasal and oral route. An auto-static holder for the endoscopes (Microfrance, Medtronic-Xomed, Jacksonville, Fla.) was mounted on the table and adjusted to the endoscope, facilitating a four-handed technique. No lidocaine and epinephrine injections were administered. The orifice of the tube was visualized with a $30^{\circ}$ angled view endoscope (4 $\mathrm{mm}$ in diameter) and the medial cartilaginous lamina and valve palpated with a curved ball tipped instrument. After identifying these structures, a KTP $532 \mathrm{~nm}$ fiber-delivered laser (Laserscope, San Jose, California, USA) was then introduced through the nose into the nasopharynx and positioned toward the diseased area of the mucosa to be ablated in the medial cartilaginous lamina within the posterior cushion. An endostat single-use fiberoptichandpiece designed for handheld surgery on very small delicate structures was used housing a $0.6 \mathrm{~mm}$ fiber. The settings were 2 - 4 watts continuously. Laser ablation of the mucosa was begun over the medial edge of the posterior cushion where a mucosal defect was created starting in the center ablating most mucosa on the anterior surface of the posterior cushion. Laser ablation of the submucosal tissue and perichondrium was also accomplished until the cartilaginous superstructure of the posterior cushion was identified and discovered creating a defect from the proximal to distal portion into the tubal lumen to a point 3 - $4 \mathrm{~mm}$ from the functional valve. The valve area was not touched with the laser. Mild, moderate and severe obstructive conditions were treated differently.

The shape and size of the area to be ablated was assessed preoperatively depending on the stage of COETD. In all cases, ChEt was applied to the ET cartilage frame as proposed by the authors. Half or full thickness incisions were made on the concave side of the cartilaginous superstructure of the posterior cushion and extended as close as possible toward the valve area. The opposite mucoperichondrium was not reached. This resulted in a far more effective release of inherent interlocked forces in the cartilage and more reliable long term straightening favoring better TVPM mobility. 
No ET packing or stents were used at the end of the procedure. Care was taken not to injure the mucosa of the anterior edge of the ET to avoid scar band formation. Patients were discharged from the hospital on the same day of the surgical procedure and were instructed to gently blow their noses and use hypertonic saline solution for rinsing their noses. All patients received postoperative nasal steroids and oral dexametasone at a dose of 40 mg daily for 5 days. Both were started on day 2 and were suspended on day 8, postoperatively. Immediate postoperative control visits took place two and four weeks later and long term follow-up was done in all 120 patients at a year, two, and five years, postoperatively. In the postoperative visits we made the 3-point scale symptom recording tool, auditory battery tests (tone audiometry, tympanometry, ET-tympanometric tests) the SMVEA and compared to the preoperative data.

\subsection{Statistical Method of Analysis}

All patient data and findings were recorded. The data was transferred into a microcomputer and analyzed using the StatPac statistical software program (Bloomington, MN). Symptom scores were estimated for every preoperative, those at one and four weeks, one, two and five years postoperative symptoms and analyzed using the Wilcoxon's test with one-tailed interpretation. Wilcoxon's two-tailed test was used for analyzing the pre and postoperative simple endoscopic findings and slow-motion endoscopic analysis data and also when correlations of ET patency on pre and postoperative differences in symptoms and findings were tested. In cases of bilateral symptoms, only the worst ET was used in the analyses.

\section{Results}

Analysis of data obtained from preoperative CT scans of the ear showed the following: normal shape and width $(1.3 \mathrm{~mm}-3.5 \mathrm{~mm})$ of the bony portion of the ET in all 120 patients $(100 \%)$. No bony dehiscence were recognized in the carotid canal and no opacification was seen in the bony portion. The intraluminal ET mucosa was preoperatively classified as seen on CT scans as normal in $2 \%$ of the patients and hypertrophic in $98 \%$.

According to the Yañez and Mora Classification System [6] we found 8 patients (7\%) with Stage 0 COETD (score $<13) 14$ patients (12\%) with Stage 1 COETD with a score $<22$ indicating mild COETD. 28 patients (23\%) had Stage 2 (score < 34) indicating moderate COETD. 70 patients (58\%) had Stage 3 disease (score < 39) indicating severe COETD. Table 1 shows the classification system corresponding to the surgical plan on each patient.

Comparing the data of slow motion video endoscopic analysis, before surgery half of the patients had moderate impairment of valve aperture, and the other half between mild and severe impairment. Mobility of the medial aspect of the ET seen during SMVEA improved notoriously the first year in 87\% of the cases (105 patients) with good valve dilation. Fifteen patients (13\%) had a moderate improvement in ET mobility. At five years postoperatively, 118 of the 120 patients had normal middle ears and their tympanic membranes looked well and also demonstrate a good valve dilation on SMVEA (Figure 2). One patient had another episode of middle ear effusion in one ear and required a third tympanostomy tube placement. A titanium middle ear grommet was inserted for permanent ventilation.

\section{Table 1. Stages of chronic obstructive Eustachian tube disease and laser ablation extension.}

Disease stage
1 Ablension of laser ablation in the Eustachian tube
cushion. Oval shaped ablation defect is centered over this cartilage. Perichondrium is not ablated.
Mucosa and submucosal tissue (perichondrium) overlying the leading edge of the lateral cartilaginous
lamina and the posterior cushion oval shaped ablation defect is centered over this cartilage only.
No ablation is carried out distally to the Eustachian tube valve.
Mucosa, submucosal, perichondrium and cartilage are thinned. Effort is made to weaken the spring
of the cartilage. Oval shaped ablation defect is centered over this cartilage only. Medium thickness
cross-hatching of the PC tissue ablation is extended distally to within 4 - 5 mm of the Eustachian tube valve.
Mucosa, submucosal, perichondrium and cartilage are thinned. Effort is made to weaken the spring of the
cartilage. Oval shaped ablation defect is centered over this cartilage and extended distally to within
4 - 5 mm to adjacent structures. Full thickness cross-hatching of the posterior cushion.




\section{SLOW MOTION VIDEO ENDOSCOPY}

BEFORE SURGERY

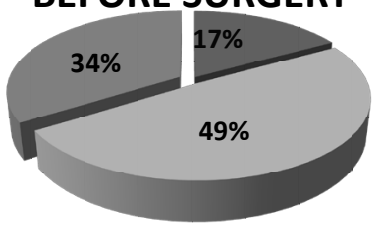

$\mathbf{n = 1 2 0} \quad$ MILD $\square$ MODERATE $\square$ SEVERE

\section{SLOW MOTION VIDEO ENDOSCOPY ANALYSIS AT 1 YEAR POSTOPERATIVELY}

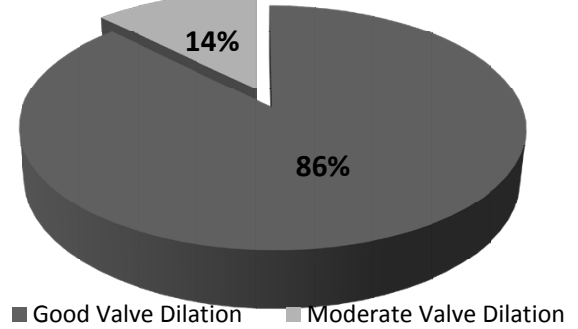

SLOW MOTION VIDEO ENDOSCOPY ANALYSIS AT 5 YEAR POSTOPERATIVELY

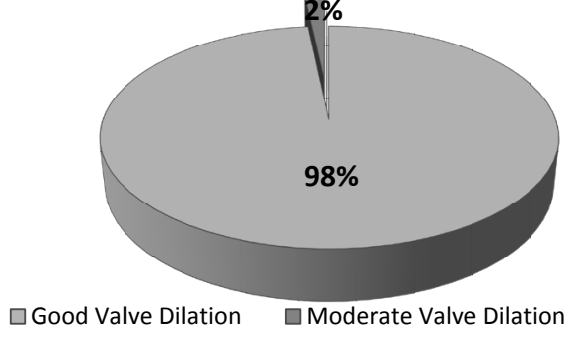

Figure 2. Before surgery $49 \%$ had moderate valve impairment. After the first year $87 \%$ of patients had good valve dilation, at the end of the study $98 \%$ reach good valve dilation. Slow motion video endoscopy analysis before surgery, 1 year and 5 years after the procedure. $\mathrm{n}=$ number of patients.

Intraoperative anatomical variations of the ET most commonly found included severely malformed posterior cushion cartilage in 28 patients (23.33\%) with severe obstruction of the functional valve area. Obstructive hypertrophy of the TVPM, LVPM and SphM areas was seen in 35 patients (29.16\%). Remarkable mucosal hypertrophic disease of the PC was seen in 31 patients (25.83\%), and in 16 patients (13.33\%) recorded in the vicinity of the functional valve area. Severe scarring tissue completely occluding the proximal tubal lumen and partially obscuring the PC was seen in one patient $(0.83 \%)$ the remaining 9 had polypoid obstruction

Ninety three patients (77.5\%) were seen after a week. The rest (27 patients) $22.5 \%$ were unable to return for their postoperative visit because they lived distant from the city. These patients did not refer any complaints when spoken to on the telephone. One hundred and twelve patients $(93.3 \%)$ did go to the $1^{\text {st }}$ year postoperative visit and 120 patients (100\%) did go to the $2^{\text {nd }}$, and $5^{\text {th }}$ year follow-up examination.

\subsection{Pre and Postoperative Symptoms}

A symptom severity product (SSP) value was estimated for the 3-point scale symptom preoperatively, at one, two and five years postoperatively during follow-up and carefully compared. We observed how symptoms decrease during the follow up, being minimal at 5 years postoperatively (Figure 3).

\subsection{Tympanometry Results}

ET tympanometric tests were carried out in all patients at 1 year, $2^{\text {nd }}$ and $5^{\text {th }}$ year postoperative and compared to the preoperative data, we included 198 Eustachian tubes. Before Surgery we found 183 Type B Tympanometries, after the first year 176 were Type A curves and for the 5th year 185 were Type A curves (Figure 4).

\section{Discussion}

Cross-hatching Eustachian Tuboplasty is a relatively new procedure [5], careful patient selection criteria must be established. A lot has been written about Eustachian tube dysfunction and several procedures have been proposed in the last years [7]. A comparison of the studies assessing the efficacy of Eustachian tube surgery is often difficult due to the lack of a common staging system or a proper definition of COETD. There is a recent and promising classification proposed as a novel diagnostic tool [8], in this study, all eligible patients were studied 


\section{Symptom Severity Product (SSP) Values for Eustachian Tube Obstructive Dysfunction Preoperative and follow up data $\mathrm{N}=120$ Patients}

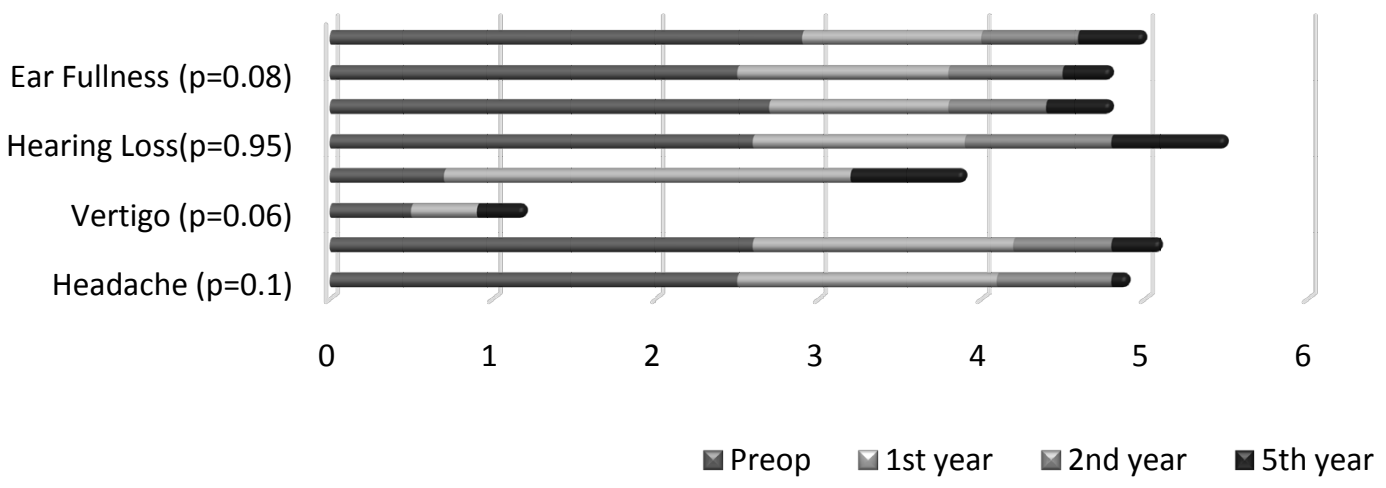

Figure 3. A symptom severity product (SSP) value was estimated for the 3-point scale. We can see that the symptoms trend to disappear at the end of the study.

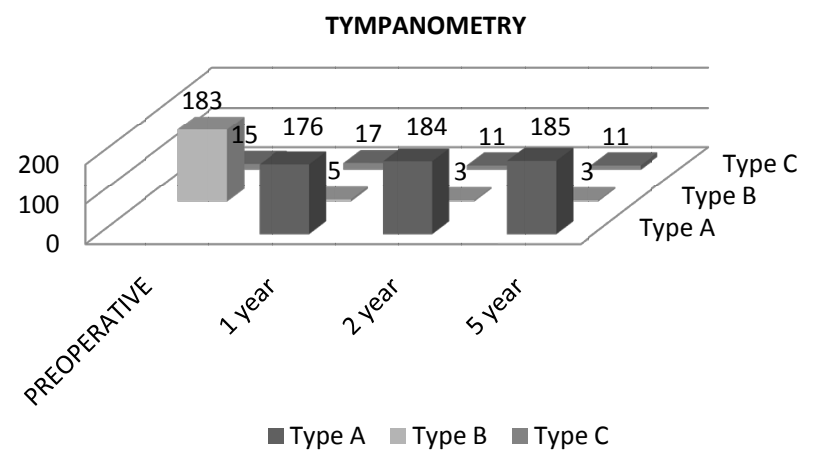

Figure 4. We include 198 Eustachian tubes. Preoperative there were 183 curve B Tympanometries, in the first year 176 were $A$ and for the $5^{\text {th }}$ year 185 were A curves.

using the classification system we proposed in 2008 [6].

The intraluminal ET mucosa on the CT scan was normal in $2 \%$ of the patients and hypertrophic in $98 \%$, however, it is important to consider that often times X-rays, CT scans and other procedures produce considerably false negative results when edema, opacification and polyps occur and assessed in the ET lumen. CT scans are more useful when assessing the status of the isthmus and the bony ET portion.

Before surgery $93 \%$ of patients had Eustaquian tube disease, regarding our classification system, at the end of the study we reach $99 \%$ of patients without Eustachian tube dysfunction (Stage 0) the remaining 1\% were patients that developed associate conditions (laryngo pharyngeal reflux, allergic rhinitis).

In the slow motion video endoscopy before surgery we found $49 \%$ of the patients with moderate impairment of valve aperture, and 51\% between mild and severe impairment. At five years postoperatively, we can appreciate in $98 \%$ the improvement of valve dilation.

The improvement of symptoms was measured at 1,2 and 5 years after surgery and compared with the preoperative data, having a $96 \%$ of patients without symptoms at the end of the study.

Although, the patient sample size is sufficient for drawing conclusions from the different outcomes in this report, large numbers of patients are still needed in more detailed studies in the near future. Correct diagnosis of COETD is of crucial importance in planning the surgical treatment of the chronic disease. Endoscopic methods have improved the diagnostic precision, the use of SMVEA is of the most important. In this study, SMVEA was the tool for reaching a detailed diagnosis of COETD and valve dilation capability.

The major drawback, in an attempt to quantify disease extension, is that repeated ET endoscopies for SMVEA may be considered painful or cause severe discomfort to patients. No other test for measuring ET function postoperatively is as reliable. 
Additional useful information is obtained from the patient's history. In order to adequately choose the correct surgical approach, a staging system is helpful. Until now, there are no standardized criteria for the selection of cases that are appropriate for the ChEt procedure mainly because of differences in surgical techniques favored by each surgeon. According to our stage system, ChEt is recommended in COETD Stages 2, 3 and 4. However, a cautious interpretation of endoscopic findings is needed to compare the treatment results because of various extensions of the disease and surgical treatment. To clarify the indication of a ChEt approach in the future, the recurrence rate should be analyzed according to the stage of COETD. Therefore, preoperative staging is highly important. This will increase the choice of the adequate surgical ET tissue ablation extension and approach.

The Balloon dilatation of the Eustachian tube surgery [9] [10] is another technique for treating Eustachian tube dysfunction, it has been published a clear short term (6 months) benefit across all recorded outcome measures in a majority of cases, with symptom improvement in $67 \%$ of cases at 2 months.

We think we have to compare both techniques and large numbers of patients are still needed to reproduce results.

\section{Conclusion}

These outcome results suggest that ChEt is safe and efficacious in the treatment of COETD. Preoperative staging of COETD is useful for planning an appropriate surgical approach. The numerical score system used here can objectively quantify and provide a high level of agreement in the evaluation of COETD. We recommend it for reproducing it in ET surgery in large clinical studies. Further evaluation of longer term follow-up will be necessary to determine whether these patients achieve or continue to have good results.

\section{References}

[1] Hopf, J., Linnarz, M., Gundlach, P., et al. (1991) Die Mikroendoskopie der eustachischen rohre und des mittelhres: Indikationen und klinischer einsatzpunkt. Laryngorhinootologie, 70, 391-394. http://dx.doi.org/10.1055/s-2007-998060

[2] Kujawski, O. (2000) Laser Eustachian Tuboplasty (LEPT): $4^{\text {th }}$ European Congress of EUFOS. Otolaryngology—Head and Neck Surgery, 2, 835-842.

[3] Kujawski, O.B. and Poe, D.S. (2004) Laser Eustachian Tuboplasty. Otology Neurotology, 25, 1-8. http://dx.doi.org/10.1097/00129492-200401000-00001

[4] Poe, D.S., Metson, R.B. and Kujawski, O. (2003) Laser Eustachian Tuboplasty: A Preliminary Report. Laryngoscope, 113, 583-591. http://dx.doi.org/10.1097/00005537-200304000-00001

[5] Yañez, C. (2010) Cross-Hatching Technique for Eustachian Tuboplasty: Preliminary Report. Otolaryngology—Head and Neck Surgery, 142, 688-693. http://dx.doi.org/10.1016/j.otohns.2009.12.046

[6] Yañez, C. and Mora, N. (2008) Classification System for Results in Eustachian Tube Surgery. Otolaryngology—Head and Neck Surgery, 139, P74. http://dx.doi.org/10.1016/j.otohns.2008.05.238

[7] McCoul, E.D., Lucente, F.E. and Anand, V.K. (2011) Evolution of Eustachian Tube Surgery. The Laryngoscope, 121, 661-666.

[8] Schröder, S., Lehmann, M., Sauzet, O., Ebmeyer, J. and Sudhoff, H. (2014) A Novel Diagnostic Tool for Chronic Obstructive Eustachian Tube Dysfunction-The Eustachian Tube Score. The Laryngoscope. http://dx.doi.org/10.1002/lary.24922

[9] Miller, B.J. and Elhassan, H.A. (2013) Balloon Dilatation of the Eustachian Tube: An Evidence Based Review of Case Series for Those Considering Its Use. Clinical Otolaryngology, 38, 525-532. http://dx.doi.org/10.1111/coa.12195

[10] Poe, D.S., Silvola, J. and Pyykko, I. (2011) Balloon Dilation of the Cartilaginous Eustachian Tube. OtolaryngologyHead and Neck Surgery, 144, 563-569. http://dx.doi.org/10.1177/0194599811399866 
Scientific Research Publishing (SCIRP) is one of the largest Open Access journal publishers. It is currently publishing more than 200 open access, online, peer-reviewed journals covering a wide range of academic disciplines. SCIRP serves the worldwide academic communities and contributes to the progress and application of science with its publication.

Other selected journals from SCIRP are listed as below. Submit your manuscript to us via either submit@scirp.org or Online Submission Portal.
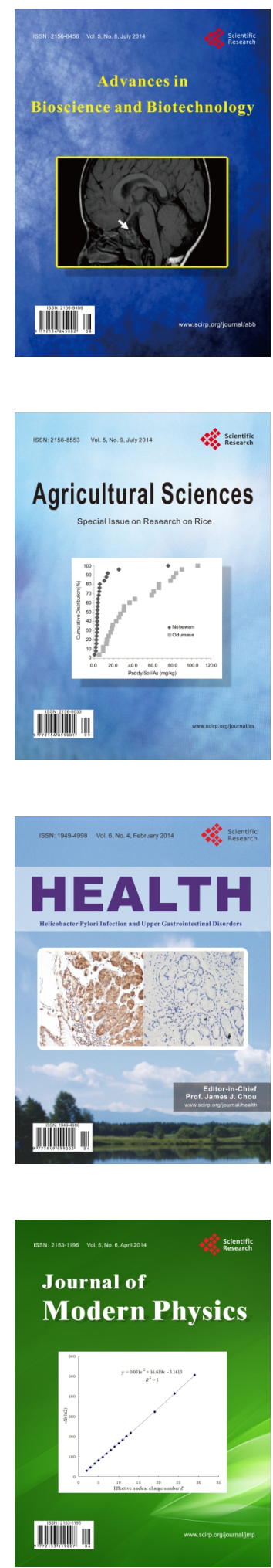
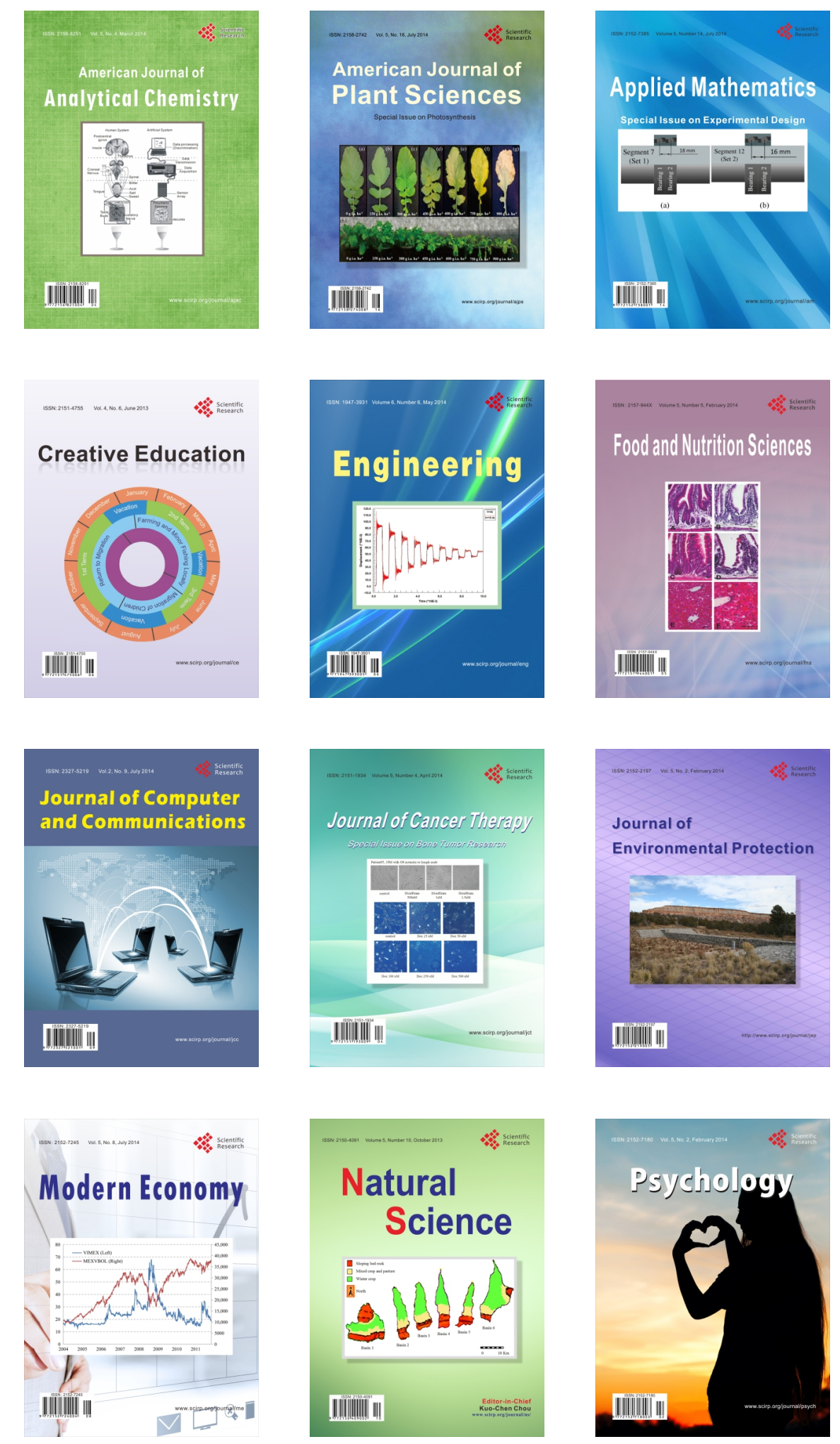